\title{
A Comprehensive Evaluation Model for Remote Sensing Fused Image
}

\author{
Jiang-Long $\mathrm{Wu}^{1, \mathrm{a}^{*}}$ and Xiao-Lin Tian ${ }^{2, \mathrm{~b}}$ \\ ${ }^{1}$ Faculty of Information Technology, Macau University of Science and Technology, Macao, China \\ ${ }^{2}$ Lunar and Planetary Science Laboratory, Space Science Institute, Macao, China

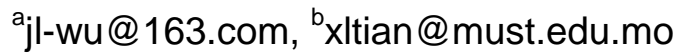

Keywords: comprehensive evaluation metric; PCNN; RPCA; remote sensing.

Abstract. This paper presents a novel evaluation model for remote sensing fused images. Meanwhile, take IHS-RPCA and NSCT-SF-PCNN as an example, the simulation experiments are analyzed and measured by the proposed comprehensive evaluation metric model. In addition, we brief introduce the choice for the weight $\omega$.Through this study we conclude that the proposed evaluation model is valid for the measure for remote sensing fused images.

\section{Introduction}

Image fusion is an image processing method that can be described as combining information from images of different sources or modalities into a composite image.

The fusion of multispectral (MS) and panchromatic (PAN) images, with complementary spectral and spatial characteristics, is becoming a promising technique to obtain images with high spatial and spectral resolution simultaneously. Due to the advances in satellite technology, a great amount of image data has been available and widely used in different remote sensing applications, such as feature detection, change monitoring, urban analysis, and land cover classification etc. Thus, image data fusion has become a valuable tool in remote sensing to integrate the best characteristics of each sensor data involved in the processing.

At present, there are many fusion rules, such as discrete cosine transform (DCT) [1], pyramid transforms [2], multiscale geometric transforms [3], and wavelets transform (WT) [4] etc. However, how to measure the quality of fusion rules is still an important issue.

Image quality assessment (IQA) aims to use computational models to measure the image quality consistently with subjective evaluations. Since the subjective IQA methods are easily influenced by many factors including viewing distance, display device, lighting condition, subjects' vision ability, and subjects' mood etc. Therefore, it is necessary to design mathematical models that are capable of predicting the quality evaluation of an average human observer.

In this paper, we will establish an evaluation model of full reference (FR) with weight, and take remote sensing fused images as examples. The evaluation model can be mathematically described as the following:

$$
\mathscr{C}_{\mathrm{FAB}}=\omega_{\mathrm{FA}} \mathscr{E}_{\mathrm{FA}}+\omega_{\mathrm{FB}} \mathscr{E}_{\mathrm{FB}}
$$

where $\mathscr{C}_{\mathrm{FAB}}$ denotes the comprehensive evaluation metric of fused image $F$ and reference images $A$ and $B ; \mathscr{E}_{\mathrm{FX}}$ denotes the relative evaluation metric of fused image $F$ and reference image $X(X=A$, $B) ; \omega_{\mathrm{FX}}$ denotes the weight of $\mathscr{E}_{\mathrm{FX}}(X=A, B)$ in comprehensive evaluation and satisfies $\omega_{\mathrm{FA}}+\omega_{\mathrm{FB}}$ $=1$. In the following, let $A=\mathrm{MS}, B=\mathrm{PAN}, \omega=\omega_{\mathrm{FA}}$.

\section{Simulation Experiments}

In this section, we will perform simulation experiments using NSCT-SF-PCNN fusion [5] and IHS Combined Robust PCA transformation fusion [6], respectively. The results for simulation experiments are separately shown in the Fig. 1(a) and Fig. 1(b). 


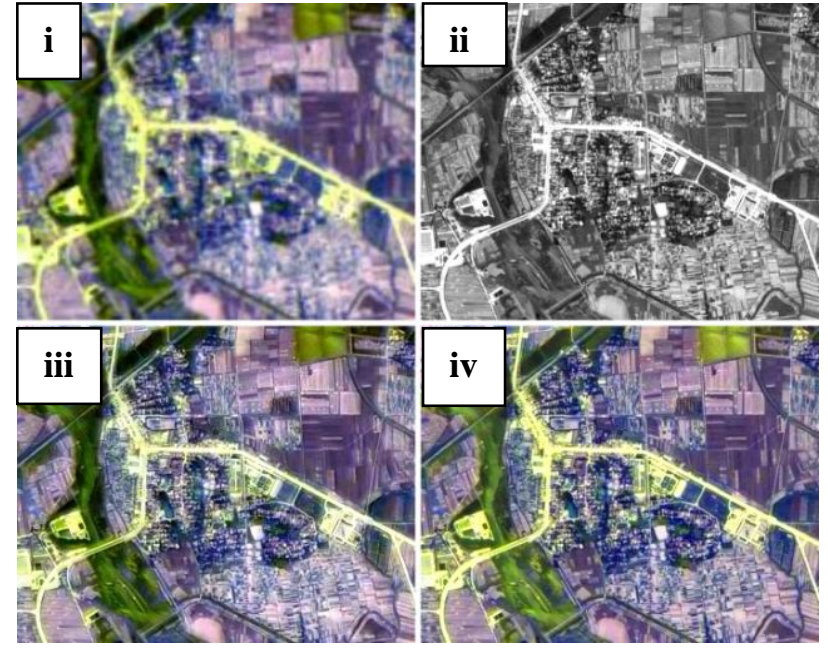

(a) (i) MS, (ii) PAN, (iii) NSCT-SF-PCNN fusion, (iv) IHS-RPCA fusion
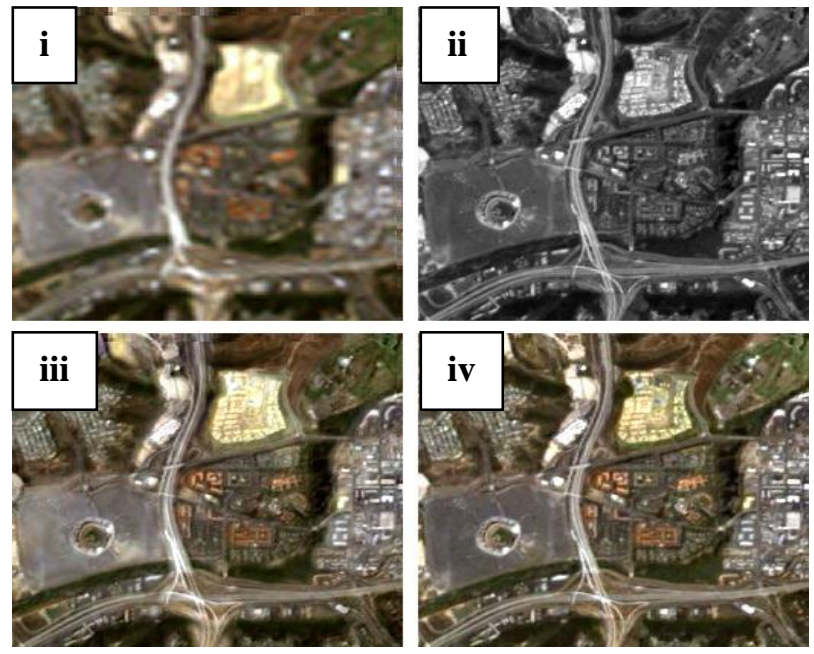

(b) (i) MS, (ii) PAN, (iii) NSCT-SF-PCNN fusion, (iv) IHS-RPCA fusion

Fig. 1: Simulation Experiments

\section{Image Evaluation and Data Analysis}

Visual comparison indicates that IHS combined RPCA transform fusion show better visual interpretability compared to the NSCT-SF-PCNN fusion techniques (see Fig. 1(a) and Fig. 1(b)).

\begin{tabular}{|c|c|c|c|c|c|c|c|}
\hline \multirow[t]{2}{*}{ Statistics } & \multirow[t]{2}{*}{ Technique } & \multicolumn{3}{|c|}{ Bands for $\mathscr{E}_{\mathrm{FA}}$} & \multicolumn{3}{|c|}{ Bands for $\mathscr{E}_{\mathrm{FB}}$} \\
\hline & & 1 & 2 & 3 & 1 & 2 & 3 \\
\hline \multirow[t]{2}{*}{$\mathrm{CC}$} & $\mathrm{NSCT}+\mathrm{PCNN}$ & 0.9375 & 0.9381 & 0.9375 & 0.9267 & 0.9302 & 0.9243 \\
\hline & IHS+RPCA & 0.9203 & 0.9197 & 0.9203 & 0.9315 & 0.9280 & 0.9338 \\
\hline \multirow[t]{2}{*}{ SSIM } & $\mathrm{NSCT}+\mathrm{PCNN}$ & 0.9450 & 0.9440 & 0.9500 & 0.9228 & 0.9254 & 0.9190 \\
\hline & IHS+RPCA & 0.9118 & 0.9129 & 0.9056 & 0.9352 & 0.9327 & 0.9387 \\
\hline \multirow[t]{2}{*}{ MI } & $\mathrm{NSCT}+\mathrm{PCNN}$ & 0.9604 & 0.9622 & 0.9531 & 0.9051 & 0.9215 & 0.9092 \\
\hline & IHS+RPCA & 0.8916 & 0.8890 & 0.9017 & 0.9504 & 0.9364 & 0.9472 \\
\hline \multirow[t]{2}{*}{ RMSE } & $\mathrm{NSCT}+\mathrm{PCNN}$ & 0.9068 & 0.9072 & 0.9172 & 0.9670 & 0.9186 & 0.9483 \\
\hline & IHS+RPCA & 0.9491 & 0.9488 & 0.9403 & 0.8816 & 0.9391 & 0.9078 \\
\hline \multirow[t]{2}{*}{ Warp } & $\mathrm{NSCT}+\mathrm{PCNN}$ & 0.9003 & 0.9007 & 0.9119 & 0.9667 & 0.9130 & 0.9484 \\
\hline & IHS+RPCA & 0.9541 & 0.9538 & 0.9449 & 0.8820 & 0.9440 & 0.9076 \\
\hline
\end{tabular}

Table 1: Evaluation Metric $\mathscr{E}_{\mathrm{FA}}$ and $\mathscr{E}_{\mathrm{FB}}$ for Fig. 1(a)

To objective evaluate the ability of enhancing spatial details and preserving spectral information, we will utilize the comprehensive evaluation metric defined by equation (1).

At first, we give some statistical parameters, such as correlation coefficient (CC), structural similarity index method (SSIM)[7], mutual information (MI) [8], root mean squared error (RMSE), and spectral distortion (warp) (see Table 1 and Table 2). For the metrics CC, SSIM and MI, a larger value means a better performance, whereas a smaller value implies better performance for RMSE and warp. Then, let $\omega \in(0,1)$, using Table 1 and equation (1), we can obtain the following Fig. 2(a). Meanwhile, similar the Fig. 2(a), applying Table 2 and equation (1), we also have the Fig. 2(b).

From Fig. 2(a) and Fig. 2(b), it is not difficult to found that the comprehensive evaluation metric $\mathscr{C}_{\text {FAB }}$ of IHS-RPCA are monotonically decreasing as $\omega$ increasing in statistical parameters CC, SSIM 
and MI, but that are monotonically increasing in statistical parameters RMSE and Warp. Whereas the comprehensive evaluation metric $\mathscr{C}_{\mathrm{FAB}}$ of NSCT-SF-PCNN are just different from IHS-RPCA, that are monotonically increasing in CC, SSIM and MI, and monotonically decreasing in RMSE and Warp.

\begin{tabular}{|c|c|c|c|c|c|c|c|}
\hline \multirow[t]{2}{*}{ Statistics } & \multirow[t]{2}{*}{ Technique } & \multicolumn{3}{|c|}{ Bands for $\mathscr{E}_{\mathrm{FA}}$} & \multicolumn{3}{|c|}{ Bands for $\mathscr{E}_{\mathrm{FB}}$} \\
\hline & & 1 & 2 & 3 & 1 & 2 & 3 \\
\hline \multirow[t]{2}{*}{$\mathrm{CC}$} & NSCT+PCNN & 0.9033 & 0.9020 & 0.8918 & 0.8554 & 0.8542 & 0.8563 \\
\hline & IHS+RPCA & 0.8619 & 0.8581 & 0.8521 & 0.8795 & 0.8792 & 0.8801 \\
\hline \multirow[t]{2}{*}{ SSIM } & $\mathrm{NSCT}+\mathrm{PCNN}$ & 0.9211 & 0.9174 & 0.9014 & 0.8551 & 0.8541 & 0.8502 \\
\hline & IHS+RPCA & 0.8602 & 0.8549 & 0.8364 & 0.8804 & 0.8795 & 0.8869 \\
\hline \multirow[t]{2}{*}{ MI } & NSCT+PCNN & 0.9332 & 0.9284 & 0.9085 & 0.8046 & 0.7884 & 0.8092 \\
\hline & IHS+RPCA & 0.8405 & 0.8389 & 0.8286 & 0.8981 & 0.8909 & 0.9045 \\
\hline \multirow[t]{2}{*}{ RMSE } & $\mathrm{NSCT}+\mathrm{PCNN}$ & 0.7990 & 0.8035 & 0.8165 & 0.9104 & 0.9430 & 0.9411 \\
\hline & IHS+RPCA & 0.8829 & 0.8911 & 0.9100 & 0.8451 & 0.8237 & 0.8055 \\
\hline \multirow[t]{2}{*}{ Warp } & $\mathrm{NSCT}+\mathrm{PCNN}$ & 0.7931 & 0.7988 & 0.8115 & 0.8880 & 0.9219 & 0.9457 \\
\hline & IHS+RPCA & 0.8831 & 0.8923 & 0.9147 & 0.8619 & 0.8424 & 0.8038 \\
\hline
\end{tabular}

Table 2: Evaluation Metric $\mathscr{E}_{\mathrm{FA}}$ and $\mathscr{E}_{\mathrm{FB}}$ for Fig. 1(b)

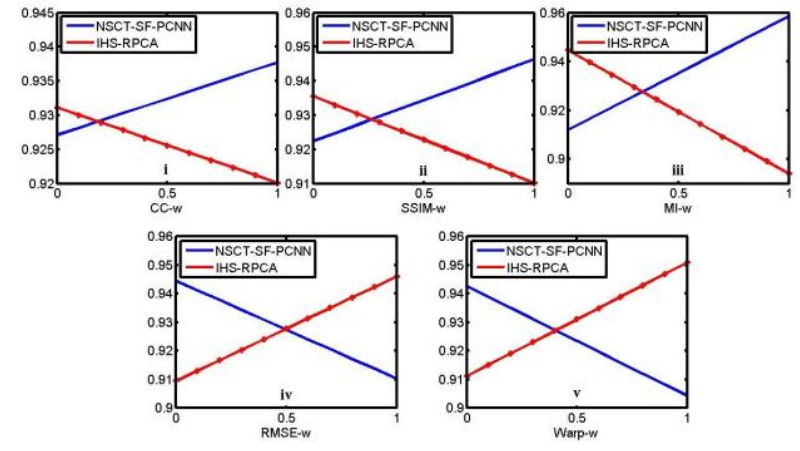

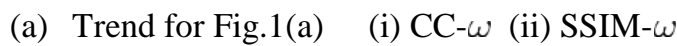
(iii) MI- $\omega$ (iv) RMSE- $\omega$ (v) Warp- $\omega$

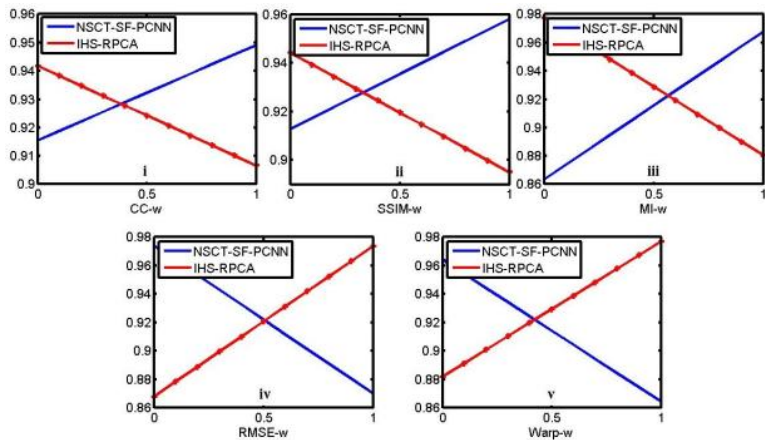

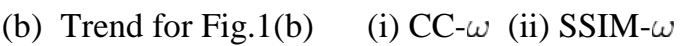
(iii) MI- $\omega$ (iv) RMSE- $\omega$ (v) Warp- $\omega$

Fig. 2: Trend of Comprehensive Indexes for NSCT-SF-PCNN \& IHS-PRCA

\begin{tabular}{ccccccc}
\hline$\overline{\mathscr{E}}_{\text {FAB }}$ & Technique & CC & SSIM & MI & RMSE & Warp \\
Fig. 1(a) & NSCT+PCNN & 0.9303 & 0.9296 & 0.9259 & 0.9344 & 0.9312 \\
& IHS+RPCA & 0.9278 & 0.9279 & 0.9295 & 0.9205 & 0.9231 \\
Fig. 1(b) & NSCT+PCNN & 0.9190 & 0.9208 & 0.8951 & 0.9225 & 0.9142 \\
& IHS+RPCA & 0.9177 & 0.9145 & 0.9285 & 0.9003 & 0.9100 \\
\hline
\end{tabular}

Table 3: Average Value of Comprehensive Evaluation Metric $\mathscr{E}_{\mathrm{FAB}}$ for Fig. 1

The change of weight $\omega$ evidence the variation of proportion for relative evaluation metric $\mathscr{E}_{F A}$ in the comprehensive evaluation metric $\mathscr{C}_{\mathrm{FAB}}$. When $\omega=0$ or $\omega=1$, the comprehensive evaluation metric $\mathscr{C}_{\text {FAB }}$ only include $\mathscr{E}_{F B}$ or $\mathscr{E}_{F A}$, it means the main component is the high spatial resolution panchromatic image PAN in the comprehensive evaluation metric $\mathscr{C}_{\mathrm{FAB}}$, or the high spectral 
resolution image MS. To Fig. 2(a) and Fig. 2(b), it can see the horizontal coordinates for intersection points are located between 0.15 and 0.55 .Taking into account the fact that the fusion process is performed at a coarser scale, that is the low resolution MS image is brought to the geometric size of the high resolution PAN image. Thus, in order to overcome this problem and objectively evaluate image, $\omega$ will take the suitable values $(0<\omega<0.5)$ to measure the fusion algorithms. And it is easy to verify that the fusion algorithm IHS-RPCA is better than the NSCT-SF-PCNN in $\omega \in(0.15,0.45)$ (see Table 3). Meanwhile, it also show that IHS-RPCA algorithm can enhance spatial details, whereas NSCT-SF-PCNN can preserve more spectral information.

\section{Summary}

This paper presents a novel evaluation model for remote sensing fused images. Meanwhile, take IHS-RPCA and NSCT-SF-PCNN as an example, the simulation experiments are analyzed and measured by the proposed comprehensive evaluation metric model. In addition, we brief introduce the choice for the weight $\omega$.Through this study we conclude that the proposed evaluation model is valid for the measure for remote sensing fused images.

\section{Acknowledgements}

The authors would like to thank the partial support from Macau Science and Technology Development Fund (No. 059/2013/A2).

\section{References}

[1] M. B. A. Haghighat, A. Aghagolzadeh, and H. Seyedarabi, Multi-focus image fusion for visual sensor networks in dct domain, Computers \& Electrical Engineering, 37 (2011), pp.789--797.

[2] W. Wang and F. Chang, A multi-focus image fusion method based on laplacian pyramid, Journal of Computers, 6 (2011), pp. 2559--2566.

[3] J. Zhao, L. Lü, and H. Sun, A novel multi-focus image fusion method using shearlet transform, in Advanced Materials Research, Trans Tech Publ, 121(2010), pp. 373--378.

[4] X. Li, M. He, and M. Roux, Multifocus image fusion based on redundant wavelet transform, IET image processing, 4 (2010), pp. 283--293.

[5] X. Qu, J. Yan, H. Xiao, and Z. Zhu, Image fusion algorithm based on spatial frequency motivated pulse coupled neural networks in nonsubsampled contourlet transform domain, Acta Automatica Sinica, 34 (2008), pp. 1508--1514.

[6] Y. Tian, J. Wu, and X. Tian, Satellite image fusion using RPCA combined IHS transform, in Optics and Precision Engineering Forum, in press, 2015.

[7] Z. Wang, A. C. Bovik, H. R. Sheikh, and E. P. Simoncelli, Image quality assessment: from error visibility to structural similarity, Image Processing,IEEE Transactions on, 13(2004),pp.600--612.

[8] D. J. MacKay, Information theory, inference and learning algorithms, Cambridge university press, 2003. 